\title{
Anabases
}

ANABASES Traditions et réceptions de l'Antiquité

12 | 2010

Varia

\section{Sul carattere cristiano della legislazione costantiniana}

Giuliano Crifò

\section{(2) OpenEdition}

1 Journals

Edizione digitale

URL: http://journals.openedition.org/anabases/1155

DOI: 10.4000/anabases.1155

ISSN: 2256-9421

Editore

E.R.A.S.M.E.

\section{Edizione cartacea}

Data di pubblicazione: 1 ottobre 2010

Paginazione: 119-125

ISSN: 1774-4296

\section{Notizia bibliografica digitale}

Giuliano Crifò, « Sul carattere cristiano della legislazione costantiniana », Anabases [Online], 12 | 2010,

Messo online il 01 octobre 2013, consultato il 20 octobre 2019. URL : http://journals.openedition.org/ anabases/1155; DOI : 10.4000/anabases.1155

(c) Anabases 


\section{Sul carattere cristiano della legislazione costantiniana}

GiUliano CRifò

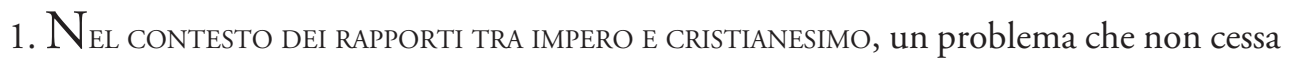
di essere affrontato riguarda il ruolo assunto da Costantino e, nella specie, una possibile lettura cristiana della sua attività normativa. Non starò qui a ripercorrere questa vicenda in tutti i suoi aspetti né d'altro canto occorre richiamare quel che in proposito si è venuto accumulando in dottrina ${ }^{1}$. Né vorrei limitarmi, in questa occasione, a ripetere quel che vado sostenendo da tempo e che l'amico e il collega di tanti anni e di tanti incontri che qui onoriamo ben conosce. Si tratterà piuttosto di qualche accenno di metodo che non manca di tener conto di indicazioni offerte dai benemeriti curatori di questa raccolta di scritti su Roma e il cristianesimo, mutamenti, strategie, creatività.

2. È ben noto che la lettura delle costituzioni imperiali consegnateci dalla tradizione teodosiana deve essere orientata dalla consapevolezza di una sua diversità, secondo che la si faccia nel contesto del Codice ovvero in quello storicamente determinato dalla loro origine: una lettura, la prima, legata ad una esplicita intenzione

1 Rinvio in specie ai contributi ed alle indicazioni presenti in A. Demant u. J. Engemann, Hrsgg., Imperator Caesar Flavius Constantinus Konstantin der Grosse, AustellungsKatalog, Mainz, 2007, bibl. 515+CD, ai colloqui e seminari collegati alla mostra di Treviri, alla messa a punto di L. De Giovanni, Istituzioni Scienza giuridica Codici nel mondo tardo antico. Alle radici di una nuova storia, Roma 2007, $175 \mathrm{ss}$. V. anche specifici rinvii nel $\mathrm{m}$. Centralità del diritto nell'esperienza della Tarda Antichità in Trent'anni di studi sulla Tarda Antichità a c. di U. Criscuolo e L. De Giovanni, Napoli, 2009, 119 ss. ed oltre, nelle note. 
normativa generale, legata invece la seconda a momenti specifici e spesso congiunturali, ad esempio per situazioni proprie di un determinato territorio dell'impero. D'altra parte, costituzioni che, in versioni rimaste extra codicem, sono ampiamente dotate di prologhi e di motivazioni, qui invece appaiono ridotte, come si sa, oltre che a squarci, dovuti anche ad una loro frammentazione nei vari titoli dei codici teodosiano e giustinianeo $^{2}$, «ad un brevissimo sunto riproducente la sola parte dispositiva, tralasciando ogni motivazione ${ }^{3}$ ".

Ora, in un recente contributo ${ }^{4}$, si legge più di una giusta osservazione nel ricercare quelle ragioni e convincimenti che nella legislazione costantiniana possono aver valso come giustificazioni morali e di cui si possa postulare il riferimento, forse, ad una "éthique ouvertement chrétienne ${ }^{5}$ ». Ebbene, mi ha colpito leggere altrove ${ }^{6}$ (e a tutt'altro proposito) la frase "mancano prove certe ma esistono indizi che conducono verso un'ipotesi sulla quale, in precedenza, nessuno ha indagato ${ }^{7}$ ». Nel nostro caso, invece, non ci si è mai stancati di indagare, come indica ad es. un lavoro di qualche anno fa, con ampia rassegna di punti di vista altrui, ma con i quali peraltro non ci si confronta, dominato come esso è da un'ottica, assolutamente unilaterale nella valutazione dei dati, che conduce a non aver dubbi e ad interpretare l'attività di Costantino a partire dal 312 come un fatto di pura missione cristiana ${ }^{8}$. Questo pregiudizio che comunque l'intera attività legislativa costantiniana ne sia stata motivata è ovviamente rifiutato anche da chi con molta buona volontà considera evidente in alcune leggi una motivazione cristiana, che per di più in taluni casi non entrerebbe in contraddizione con la tradizione da cui sono rette norme in tema di famiglia, status personale, diritto criminale, ma non può ignorare la stridente contrapposizione che se ne ha in altri

2 Un elenco di tali occorrenze, collegate alle espressioni post alia, et cetera, pars, è offerto in E. Volterra, "Il problema del testo delle costituzioni imperiali" in La critica del testo. Atti del secondo congresso internazionale della Società italiana di storia del diritto **, Firenze, 1971,1019 ss.

3 Il problema cit., p. es. 919, 1094. Non va escluso neppure che il dispositivo codicistico, anziché essere una semplice riproduzione di quello originario, dipenda da successive innovazioni della disciplina. E comunque si dovrà tener conto di una interpretazione orientata in funzione del sistema nel quale la norma si trova ora inserita. V. anche oltre, n. 16.

4 K.-L. Noetlichs, "Éthique chrétienne dans la législation de Constantin le Grand" in Le Code Théodosien. Diversité des approches et nouvelles perspectives. Études réunies par S. Crogiez-Pétrequin et P. Jaillette, EFR 412, Rome, 2009, 225 ss.

5 K.-L. Noetlichs, op. cit., 225.

6 Cf. R. BertinetTi in Il Sole 24 Ore, 14 marzo 2010, 37.

$7 \quad$ Il possibile plagio dell' Orlando di Virginia Woolf.

8 Ch. Matson Odhal, Constantine and the Christian Empire, London-New York, 2004, nelle cui pagine ricostruttive ricorre spesso questo carattere "missionario», per concludersi (284) in modo che può ben dirsi apologetico. Singolare comunque è l'assenza di letteratura, non recentissima, ma che molto ha influenzato la ricerca. V. anche le note seguenti. 
casi ${ }^{9}$. E si tenga conto di un confronto tra l'evidenza di un supposto passaggio di Costantino al cristianesimo tra il 310 e il $314^{10}$ e quella del carattere conservativo-restaurativo e non «rivoluzionario» della sua politica ${ }^{11}$, una politica retta da una «herrische Natur» di cui D. Liebs ha mostrato come essa "prägte das Recht der Christenheit vor allem im Strafrecht für viele Jahrhunderte ${ }^{12}$ », notoriamente in misura davvero poco umanitaria.

Si può dunque ben continuare a ricercare «des indices visibles, ou même vagues, qui permettraient de prouver» quel che viene proposto come «un tentativo» di formulazione di direttive etiche cristiane qualificanti i testi legislativi costantiniani e dei quali verificare anche l'effettiva applicazione ${ }^{13}$. In effetti, non solo si può ma anzi si deve, anche se la "prova» è cosa molto difficile e se non è certo da credere che una serie di indizi possa garantire altro che verosimiglianze: considerazione ovvia che potrebbe giovarsi con Momigliano dei Some Elementary Principles of Historical Method ${ }^{14}$. Ben a proposito, del resto, il Noethlichs ha posto esplicitamente un interrogativo nel titolo stesso del suo contributo.

3. Il punto diventa quello, a cui pure si fa riferimento, di una applicazione. Questa, con tutta la sua rilevanza fattuale, è da vedersi anche in rapporto ad una eventuale efficienza normativa dei testi costantiniani in età successiva e dunque anzitutto nel codice teodosiano prima ancora che in quello giustinianeo. Ed è certo che, tenendo conto del ruolo fondatore di Costantino, è anzitutto la successiva legislazione imperiale del IV secolo che può permettere di ricavare qualche considerazione con valore probatorio in ordine al problema dell'influenza cristiana: considerazione che non sia limitata al momento congiunturale e da cui si possa argomentare, per un giudizio con qualche fondamento reale, con quella consistenza storica che si dimostri capace di superare affermazioni, comunque generiche, di possibilità e che tra l'altro non dipendano da una fonte come Eusebio, per varie ragioni non innocente ${ }^{15}$.

9 Così ad es. T. J. Chiusi, "Der Einfluß des Christentums auf die Gesetzgebung Konstantins" in K. M. Girardet (Hrsg.), Kaiser Konstantin der Grosse. Historische Leistung und Rezeption in Europa Hrsg, Bonn, 2007, 55 ss.

10 K.-M. Girardet, "Konstantin und das Christentum: die Jahre der Entscheidung 310 bis 314” in GiraRdet (Hrsg.), Kaiser Konstantin der Grosse cit., 69 ss.

11 Cf. H. Brandt, "Konstantins Reformen" in Girardet (Hrsg.), Kaiser Konstantin der Grosse cit. 31 ss. nonché i molteplici riscontri offerti nel m. Centralità del diritto cit., 132 ss.

12 D. Liebs, "Konstantin als Gesetzgeber" in Girardet (Hrsg.), Kaiser Konstantin der Grosse cit., 97 ss., 103.

13 K.-L. Noetlichs, op. cit., 227.

14 Vedili nella traduzione di R. Di Donato, Gli anni di Londra in Arnaldo Momigliano nella storiografia del Novecento a c. di L. Polverini, Roma, 2006, 133 ss.

15 Non tanto per una questione di autenticità, in specie per Vita Constantini ed i materiali in essa contenuti. In proposito v. P. SILl, Testi costantiniani nelle fonti letterarie 
Sappiamo che il processo ${ }^{16}$ che ha determinato la stesura codicistica dei provvedimenti legislativi ha condotto alla eliminazione di discorsi ed espressioni per dir così valoriali, qualificanti: non solo l'occasio legis, ma anche e sostanzialmente le ragioni esplicitate dalle motivazioni della normativa stessa. Motivazioni di varia natura, anche etiche, ma non solo etiche e che, se tali, non saranno solo proprie di una etica cristiana. Ma cosa sarebbe poi questa del tutto indifferenziata etica cristiana?

Mi rifaccio qui a un notevole saggio nel quale Elena Cavalcanti ha esaminato a suo tempo "la revisione dell'etica classica nel De Civitate $D e i^{17}$ ". La compianta amica vi ha realizzato una lettura globale del De civitate reinterpretato «nella chiave etica e nella definizione cristiana de finibus bonorum et malorum ${ }^{18}$ ". Per ciò fare, ella prendeva le mosse da De Civ. XIX, 1-3 in cui, sulla scorta del de philosophia varroniano, viene proposto e commentato l'elenco di 288 diverse scuole (o formulazioni) di etica ${ }^{19}$. Quanto all'etica cristiana, sarà quella paolina dell'amore, cioè un'etica sociale della eguaglianza in Cristo? Oppure l'etica rigoristica che esclude dalla comunità chi pecchi e impone, là dove è possibile, la penitenza? $\mathrm{O}$ ancora l'etica dell'ascetismo monastico ${ }^{20}$, o quella che può risultare, forse, grazie all'attività di Costantino e certamente dopo di lui, da una filosofia pratica che sia un compromesso di etica politica e teologia ecclesiale $^{21}$ ?

(Accademia Romanistica Costantiniana, Materiali per una palingenesi delle costituzioni tardo-imperiali, 3), Milano, 1987, XVII ss., utilissimo lavoro di uno studioso sempre più compianto. Il Noetlichs si richiama all'edictum del 324 '̇ $\pi \alpha \rho \chi 1 \omega ́ \tau \alpha 1 \zeta ~ a ̉ v \alpha \tau o \lambda ı 1 \kappa o i \varsigma \zeta$

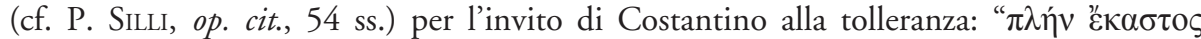

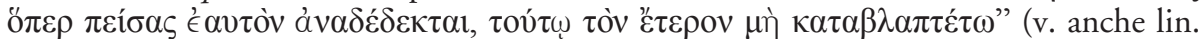

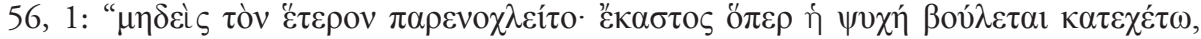

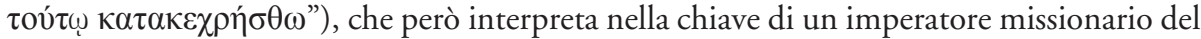
cristianesimo, dunque in chiave di etica appunto cristiana. A parte ciò, è corretta l'idea (K.-L. Noetlichs, op. cit., 226) di invertire l'ordine delle cose e cioè ricavare eventualmente la stessa autenticità della lettera da una provata influenza cristiana su documenti legislativi, certo maggiormente affidabili.

16 V. già sopra n. 2 (e, in particolare sulle ipotesi suggerite dal Volterra, v. i rilievi critici di F. WIEACKER, Römische Rechtsgeschichte I, München, 1988, 175 con le note).

17 E. Cavalcanti in Il De civitate Dei, l'opera, le interpretazioni, l'influsso a c. di E. C., Roma Freiburg-Wien, 1996, 293 ss.

18 Ivi, 323.

19 Ivi, 294 ss.; schema 1 a p. 295.

20 Indicazioni e lett. in J. RoHLs, Geschichte der Ethik, Tübingen, 1991, trad. P. Kobau, Bologna 2002, pass.

21 J. Rohls, op. cit., 126 s. Ma v. in specie R. Dillmann, Das Eigentliche der Ethik Jesu. Ein exegetischer Beitrag zur moraltheologischen Diskussion um das Proprium einer christlichen Ethik, Mainz, 1984; E. CAVAlCANTI, Etica cristiana nei secoli III e IV in L'etica cristiana nei secoli III e IV: eredità e confronti, XXIV Incontro di studiosi dell'antichità cristiana, Roma, 1966, 11 ss.; C. TruzzI, Etica dei rapporti con il divino. Pagani e cristiani nella seconda metà del IV secolo, ivi, 69 ss. 
4. È giusto dunque, per ricercarne tracce nella legislazione costantiniana, cominciare col procedere ad una sorta di tassonomia di quelli che si considerano essere valori tipicamente cristiani ${ }^{22}$, dalla fedeltà all'impegno preso con il battesimo alla astensione dai piaceri. Però, la preghiera, la carità, il perdono, l'ordine, la beneficenza, il soccorso a poveri, a orfani, a vedove ecc. non sono sentimenti e manifestazioni nati con il cristianesimo né sono tipicamente cristiani e comunque in qualche caso non han proprio a che fare con la normazione costantiniana. Per esempio, la normazione imperiale del battesimo non ha a che fare con Costantino ${ }^{23}$ né occorre immaginare particolari influenze cristiane su una sua politica normativa volta a disciplinare, in vista della sopravvivenza dei neonati, un fenomeno reso insopprimibile dalla fame ${ }^{24}$. Se poi si insiste ${ }^{25}$ su una attività costantiniana vista in cooperazione con le strutture ecclesiastiche, il giudizio deve vertere ancora sul fatto politico del sostegno costantiniano alla Chiesa ${ }^{26}$, laddove sarà solo a distanza di quasi un secolo che si renderà esplicito $\mathrm{e}$ determinante l'intervento ecclesiastico, ufficializzandone la cooperazione ${ }^{27}$.

In ogni caso, va colta questa occasione di una rinnovata verifica circa la traduzione di quell'etica in effettiva presenza, motivante la disciplina di campi rispetto ai quali la nuova religione rivendica la propria competenza. E questo alla luce di un orienta-

22 Così Noethlichs, op. cit., 225. Per questo ci si può limitare all'utile repertorio (ma le idee di fondo sono ben discutibili) di B. BIONDI, Il diritto romano cristiano I-III, Milano, 1952-54, specialmente I, 117 ss.: Orientamento cristiano dell'impero e della legislazione; II, 119 ss.: Diritto e carità, Manifestazioni della carità, Motivi etici cristiani.

23 Per tutti Biondi, op. cit., I, 1952, 161 s.

24 Cf., con riferimento in specie, per quel che ci riguarda, a Const. Th. 11.27 .1 e 2 e con valutazione che prescinde da particolari influenze cristiane, C. LORENZI, Si quis a sanguine infantem... comparaverit. Sul commercio dei figli nel tardo impero, Perugia, 2003, pass. Altri puntualissimi rilievi sulle costantiniane costituzioni di CTh.5.10.1 e 5.9.1 in ID., L'esposizione dei figli nel tardo impero. Alcune considerazioni in AARC 17, Roma, 2010, 1142 ss, $1164 \mathrm{n}$.

25 Così p. es. C. Corbo, Paupertas. La legislazione tardoantica, Napoli, 2006.

26 Costantino «era piuttosto un uomo di Stato, non esente dalla superstizione tardo-antica, che inseriva freddamente il cristianesimo nella sua politica di potenza»: H. KüNG, Cristianesimo. Essenza e storia, trad. G. Moretto, Milano, 1999, 184. Sostanzialmente conformi sono anche i commenti di vari autori in ordine agli interventi costantiniani di L. De Giovanni: cf. P.L. CarucCI, L. Di PINTO, $I l$ «Costantino» di De Giovanni. Nuove letture di una nuova edizione in SDHI LXXI, 2005, 483 ss. (m. considerazioni ivi, 499-505).

27 CTh. 5.9.2, a. 412 (cfr. C. 8.51.1, Mommsen/Krüger ad hh. 1l.; C. 8.51.2, a. 374, Biondi III, 24 n. 2). Impp. Honorius et Theodosius AA. Melitio ppo. Nullum dominis vel patronis repetendi aditum relinquimus, si expositos quodammodo ad mortem voluntas misericordiae amica collegerit, nec enim dicere suum poterit, quem pereuntem contempsit; si modo testis episcopalis subscriptio fuerit subsecuta, de qua nulla penitus ad securitatem possit esse cunctatio. V. anche l'interpretatio: Qui expositum puerum vel puellam sciente domino vel patrono misericordiae causa collegerit, in eius dominio permanebit: si tamen contestationi de collectione eius episcopus clericique subscripserint, quoniam postea suum dicere quisque non poterit, quem proiecisse probatur ad mortem. 
mento caratterizzante, che viene indicato come quello di una moralità stretta, specie, ma non solo, in tema di sessualità e che si dispiega in tema di famiglia, di matrimonio o di criminalità e più intensamente, come è chiaro, in tema di politica religiosa. Quest'ultimo è un campo in cui più attivo è naturalmente il potere politico e se si vuole la ragion di stato, in quanto determinante la misura di un regime privilegiato, in concreto per la chiesa cattolica. Ma qui ci si può limitare a dire che in questo senso la realtà di provvedimenti dove individuare uno spirito cristiano sarebbe una probabile lesione del principio ermeneutico per cui sensus efferendus, non inferendus est ${ }^{28}$.

5. I testi, ben noti e studiatissimi, si offrono a un confronto sulla lettura che ne è stata qui proposta. Essa supera la pregiudiziale di una qualche terminologia specificamente cristiana ${ }^{29}$ ed isola settori di indagine sui quali non mi pare che ci sia discordanza di opinione ${ }^{30}$. E in effetti, come ho avuto modo di dire da tempo, i cristiani e tanto più le comunità ecclesiali vivono secondo il diritto romano, al quale si adattano. Non va dimenticato, infatti, che a rigore il cristiano avrebbe dovuto rompere i legami familiari e sociali, non essere lenone, prostituta, pederasta, eunuco ecc. Ma neppure scultore, cioè idolatra, maestro di scuola, schiavo pubblico, soldato che uccide, accusatore in processi criminali, governatore di provincia, titolare dello ius gladii, funzionario con porpora, ecc; e cristiani, sì, ma non ordinati chierici, potevano essere persone ricche, agenti fiscali, macellai, fornai. Gli accomodamenti non potevano mancare ${ }^{31}$.

Dal canto suo, il Noethlichs ritiene assente nei codici un qualche provvedimento che sia testimonianza indubbia di un'etica specificamente cristiana ed afferma che in questo campo non si può andare oltre uno stato di ipotesi e di probabilità ${ }^{32}$ e poten-

28 Questo vale ad es. per l'episcopalis audientia (cfr. su Sirm. 1 Noethlichs, op. cit., 229 s.: anche a tener conto del contesto cristiano, va segnalato che la giustificazione finale non ha alcun rapporto con la religione, ma è diretta a diminuire la durata dei processi). Ed è ovvio sottolineare che privilegi e immunità sono concessioni dello stato, «tanto se essi sono in funzione dell'utilità che le situazioni privilegiate arrecano allo stato quanto se appaiano invece il risultato di una forza di pressione sul potere. Ed anche in tal caso, non va dimenticato che privilegi consimili sono attribuiti o si continuano ad attribuire anche a situazioni non cristiane, tipicamente pagane o ebraiche... A prevalere... è sempre l'interesse pubblico» (cfr. m. Lezioni di storia del diritto romano, V ed. Bologna, 2010, 470 ss.).

29 Sul punto v. le mie "Considerazioni sul linguaggio religioso nelle fonti giuridiche tardooccidentali" in Cassiodorus 5, 1999, 123 ss. nonché l'adesione, del Noethlichs (op. cit., 229 n. 5) e mia (Considerazioni cit., 130) allo scetticismo in merito manifestato in specie da P. Silli, Mito e realtà dell'aequitas christiana ecc., Milano 1980 (e adde Id., Testi costantiniani cit., sopra n. 15).

30 Cf. m. "Romanizzazione e cristianizzazione. Certezze e e dubbi in tema di rapporti tra cristiani e istituzioni" in I Cristiani e l'Impero nel IV secolo a c. di G. Bonamente, A. Nestori, Macerata, 1988, 75 ss. A titolo di esempio cfr. ivi, 98 e Noethlichs, op. cit., 233 s. su CTh. 2.25.1

31 Lezioni cit., 474 ss. Ma già, p.es., "Romanizzazione e cristianizzazione”, cit., pass.

32 Noethlichs, op. cit., 230 ss. 
zialità. Ma ciò non gli impedisce d'essere interamente d'accordo con chi congettura la presenza di elementi cristiani anche là dove è difficile - se non impossibile - darne prove assolute ${ }^{33}$. Prove, cioè - si può credere - documenti, quelli senza i quali, diceva Momigliano, "non c'è possibilità di scrivere storia ${ }^{34}$ ». Certo, si può accogliere come testimone di una coscienza etica sicuramente cristiana, versata comunque nella legislazione costantiniana, quel che racconta Sozomeno $(H E, 1.8-9)$ che di quella legislazione darebbe una descrizione oggettiva, basata su una conoscenza esatta delle leggi originali, che in origine, non avrebbero nascosto motivazioni rette da un'etica cristiana ${ }^{35}$. Non mi pare peraltro che manchino serie ragioni per dubitare di una forte attendibilità di questa fonte ${ }^{36}$. Si consideri piuttosto la legislazione postcostantiniana sugli stessi problemi di cui si è occupato Costantino, quel tema dell'efficacia a cui ho accennato in precedenza e la conferma piena che vien data del fenomeno di giuridicizzazione del cristianesimo. Ne deriva quell'assetto imperiale della chiesa e quel suo regime privilegiato che non può non porre in secondo piano una etica forte per se stessa e che possa pretendere di indirizzare il diritto nella premessa di esserne veramente distinta ${ }^{37}$.

Giuliano CRIFÒ

Università degli Studi di Roma "La Sapienza»

Piazzale Aldo Moro 5,

00185 Roma - Italia

crifo@uniromal.it

33 Noethlichs, op. cit., 237.

34 Cf. sopra, n. 9.

35 Noethlichs, op. cit., 236.

36 V., per una valutazione della qualità di Sozomeno, non come ricercatore di documenti ma come interprete di tali documenti, M. MAZZA, Sulla teoria della storiografia cristiana: osservazioni sui proemi degli storici ecclesiastici in La storiografia ecclesiastica nella tarda antichità, Messina, 1980, 335 ss., 382 ss.

37 Una formulazione adeguata di quanto qui accennato riguarda il problema del rapporto tra diritto e giustizia. Ma di questo si dirà a suo luogo. Ricerche specifiche illuminanti sono quelle ora raccolte in K.M. GIRARDET, Kaisertum, Religionspolitik und das Recht von Staat und Kirche in der Spätantike, Bonn, 2009. V. anche m. La centralità cit., passim. 